\title{
GENETIC VARIABILITY AND MULTIVARIATE ANALYSIS IN PEARL MILLET (Pennisetum glaucum (L.) R. Br.) GERMPLASM FOR DUAL PURPOSE
}

\author{
M. Shanmuganathan ${ }^{1}$, A. Gopalan ${ }^{1}$ and K. Mohanraj ${ }^{1}$
}

\begin{abstract}
Genetic variability and diversity in pearl millet (Pennisetum glaucum (L.) R.Br.) was studied with 104 germplasm accessions of different origin. Analysis of variance indicated significant variances among the accessions for all the characters studied. The maximum variation was recorded for number of tillers, number of leaves, leaf length and breadth, panicle length, leaf weight, stem weight, leaf stem ratio, green fodder yield, crude protein content and grain yield indicates the possibilities of improving these characters through phenotypic selection for the development of dual purpose cultivars (grain cum fodder yield). High heritability coupled with high genetic advance of percentage of mean was observed for all characters except days to $50 \%$ flowering suggesting that these traits are governed by additive gene action and possibility of improving these characters through selection. Based on the $D^{2}$ analysis all the accessions were grouped into twelve clusters. Cluster I retain the maximum accessions of 82 and nine clusters retained only one accession each. The intra cluster distance ranged from 0.00 to 6.71 and the inter cluster distance was recorded highest between cluster XI and cluster XII. Cluster XII exhibited maximum mean value for five characters. Based on the relative contribution, plant height contributed more towards genetic divergence.
\end{abstract}

Keywords: Pearl millet, genetic diversity, cluster distance, $D^{2}$ value, cluster mean, variability.

\section{INTRODUCTION}

Pearl millet is a stable diet for the vast majority of poor farmers and also forms an important fodder crop for livestock population in arid and semiarid regions of the country. Increased emphasis on development of dual purpose (grain cum fodder) pearl millet, is therefore necessary for ensuring high grain yield as well as higher dry fodder yield under rainfed cultivation (Dangaria and Atara, 2004).

The development of an effective breeding programme is dependent upon the existence of genetic variability. Pearl millet is endowed with a rich reservoir of genetic variability for various yield components, adaptation and quality traits (Berwal and Khairwal, 1997). Exploitation of the genetic variability in the available germplasm holds promise for producing high grain and fodder yielding hybrids. The more diverse the parents, the greater are the chances of obtaining new combinations of genes and therefore increasing the probability for crop improvement (Berwal and Khairwal, 1997). Several measures have been used to assess genetic diversity among plant populations. Of these measures, multivariate analysis provides the most reliable information. Among the multivariable procedures, Mahalanobis (1936) generalized distance $\left(\mathrm{D}^{2}\right)$ has been used extensively. Therefore, in the present investigation, an attempt has been made to assess the variability and diversity in Pearl millet germplasm 
for grain and fodder yield and its associating characters.

\section{MATERIALS AND METHODS}

One hundred and four Pearl millet accessions of diverse origin obtained from ICRISAT, Hyderabad; Department of Millet Crops and Department of Forage Crops, Tamil Nadu Agricultural University, Coimbatore were used for this study. The accessions were evaluated during Rabi 2002-2003 in a randomized block design with two replications in the Department of Forage Crops, Centre for Plant Breeding and Genetics, TNAU, Coimbatore. Each accession was raised in two rows of $2 \mathrm{~m}$ length spaced at $45 \mathrm{~cm}$ between rows and 15 $\mathrm{cm}$ between plants. The recommended agronomic practices and plant protection measures were adopted uniformly. Five randomly selected plants were taken from each replication for data recording. Data included days to $50 \%$ flowering, plant height $(\mathrm{cm})$, number of tillers per plant, number of leaves per plant, leaf length $(\mathrm{cm})$, leaf breadth $(\mathrm{cm})$, panicle length $(\mathrm{cm})$, stem diameter (mm), leaf weight (gm), stem weight (gm), leaf stem ratio (ratio between the fresh leaf weight per plant to its green stem weight), stover yield per plant (gm), crude protein content (the total nitrogen content was estimated by Humphrie's (1956) micro kjeldahl method and the crude protein content was calculated by multiplying the total nitrogen content by the factor 6.25 and was expressed in percentage) and grain yield per plant (gm). Various variability parameters were worked out following Burton (1952). The method suggested by Lush (1940) was adopted to work out the estimates of broad sense heritability. The expected genetic advance was calculated as suggested by Johnson et al. (1955). $\mathrm{D}^{2}$ values between any two accessions were estimated by multivariate analysis using Mohalanobis (1936) $\mathrm{D}^{2}$ statistic as described by Rao (1952). On the basis of $\mathrm{D}^{2}$ values, accessions were grouped into different clusters according to Tocher's method (Rao, 1952).

\section{RESULTS AND DISCUSSION}

The analysis of variance revealed highly significant differences among the accessions for all characters, indicating adequate genetic variability among the accessions. The estimates of phenotypic and genotypic variances and coefficient of variation, heritability (BS) and the genetic advance expressed at $5 \%$ selection intensity and the same expressed as per cent of mean are presented in Table 1. A wide range of variation was observed for all the characters studied. The genotypic coefficient of variation (GCV) and phenotypic co-efficient of variation (PCV) estimates ranged from 3.25 (Days to $50 \%$ flowering) to 47.78 (stem weight) and 3.54 (Days to 50\% flowering) to 48.18 (stem weight), respectively. Except days to 50\% flowering, plant height and stem diameter, all other characters showed high PCV and GCV estimates. This suggests that the selection based on these characters would facilitate successful isolation of desirable types. A similar finding was reported by Vidyadhar et al. (2004) for grain and fodder yield.

Estimation of GCV alone does not assess the amount of heritable variation. GCV computed in conjuction with heritability estimates would provide a better indication for selection on the phenotypic performance (Burton, 1952). In the present investigation, the estimates of heritability worked out in broad sense ranged from 84.37 (days to $50 \%$ 
flowering) to $99.90 \%$ (plant height). Almost all the characters had high heritability values indicating high transmission index for these characters.
Similar result was obtained by Suthamathi and Stephen Dorairaj (1995).

Table 01: The estimates of phenotypic and genotypic variances and coefficient of variation, broad sense heritability and genetic advance in pearl millet.

\begin{tabular}{|c|c|c|c|c|c|c|c|c|c|c|}
\hline \multirow{2}{*}{$\begin{array}{l}\text { S. } \\
\text { No. }\end{array}$} & \multirow{2}{*}{ Characters } & \multirow{2}{*}{ Mean } & \multirow{2}{*}{ Range } & \multicolumn{2}{|c|}{ Variance } & \multicolumn{2}{|c|}{$\begin{array}{l}\text { Co-efficient of } \\
\text { variation }\end{array}$} & \multirow[t]{2}{*}{$\begin{array}{l}\text { Heritabilit } \\
\text { y (broad } \\
\text { sense) } \%\end{array}$} & \multirow[t]{2}{*}{$\begin{array}{l}\text { Genetic } \\
\text { advance }\end{array}$} & \multirow{2}{*}{$\begin{array}{c}\text { Genetic } \\
\text { Advance } \\
\text { as \% of } \\
\text { mean }\end{array}$} \\
\hline & & & & $\begin{array}{l}\text { Phenot } \\
\text { ypic }\end{array}$ & Genotypic & $\begin{array}{c}\text { Phenotypi } \\
\text { c }\end{array}$ & Genotypic & & & \\
\hline 1. & $\begin{array}{l}\text { Days to } \\
50 \% \\
\text { flowering }\end{array}$ & 52.66 & $\begin{array}{c}48.50- \\
57.00\end{array}$ & 3.47 & 2.93 & 3.54 & 3.25 & 84.37 & 3.24 & 6.15 \\
\hline 2 & $\begin{array}{l}\text { Plant height } \\
(\mathrm{cm})\end{array}$ & 164.25 & $\begin{array}{l}56.65- \\
233.60\end{array}$ & $\begin{array}{c}947.6 \\
5\end{array}$ & 946.71 & 18.74 & 18.73 & 99.90 & 63.35 & 38.57 \\
\hline 3. & $\begin{array}{l}\text { Number of } \\
\text { tillers }\end{array}$ & 5.24 & $\begin{array}{l}2.50- \\
10.75\end{array}$ & 2.85 & 2.76 & 32.22 & 31.74 & 97.05 & 3.37 & 64.41 \\
\hline 4. & $\begin{array}{l}\text { Number of } \\
\text { leaves }\end{array}$ & 34.42 & $\begin{array}{l}15.70- \\
78.50\end{array}$ & $\begin{array}{c}155.5 \\
9\end{array}$ & 154.41 & 36.24 & 36.10 & 99.24 & 25.50 & 74.08 \\
\hline 5. & $\begin{array}{l}\text { Leaf length } \\
(\mathrm{cm})\end{array}$ & 47.45 & $\begin{array}{l}21.75- \\
66.95\end{array}$ & 91.93 & 90.96 & 20.21 & 20.10 & 98.94 & 19.54 & 41.19 \\
\hline 6. & $\begin{array}{l}\text { Leaf } \\
\text { breadth } \\
(\mathrm{cm})\end{array}$ & 3.14 & $\begin{array}{l}1.45- \\
5.45\end{array}$ & 0.60 & 0.55 & 24.55 & 23.63 & 92.67 & 1.47 & 46.87 \\
\hline 7. & $\begin{array}{l}\text { Panicle } \\
\text { length }\end{array}$ & 20.64 & $\begin{array}{l}7.95- \\
36.55\end{array}$ & 33.54 & 33.15 & 28.07 & 27.90 & 98.83 & 11.79 & 57.14 \\
\hline 8. & $\begin{array}{l}\text { Stem } \\
\text { diameter } \\
(\mathrm{mm})\end{array}$ & 8.55 & $\begin{array}{l}5.62- \\
11.71\end{array}$ & 1.70 & 1.68 & 15.26 & 15.15 & 98.68 & 2.65 & 31.01 \\
\hline 9. & $\begin{array}{l}\text { Leaf weight } \\
\text { (gm) }\end{array}$ & 55.51 & $\begin{array}{l}23.10- \\
163.05\end{array}$ & $\begin{array}{c}494.6 \\
0\end{array}$ & 493.87 & 40.07 & 40.04 & 99.85 & 45.75 & 82.41 \\
\hline 10. & $\begin{array}{l}\text { Stem } \\
\text { weight } \\
(\mathrm{gm})\end{array}$ & 235.74 & $\begin{array}{l}51.85- \\
742.05\end{array}$ & $\begin{array}{l}1290 \\
0.49\end{array}$ & $\begin{array}{c}12686.8 \\
4\end{array}$ & 48.18 & 47.78 & 98.34 & 230.10 & 97.61 \\
\hline 11. & $\begin{array}{l}\text { Leaf stem } \\
\text { ratio }\end{array}$ & 0.26 & $\begin{array}{l}0.13- \\
0.63\end{array}$ & 0.01 & 0.01 & 35.24 & 35.06 & 98.98 & 0.19 & 71.85 \\
\hline 12. & $\begin{array}{l}\text { Stover yield } \\
(\mathrm{gm})\end{array}$ & 291.25 & $\begin{array}{l}84.20- \\
857.65\end{array}$ & $\begin{array}{l}1739 \\
2.19\end{array}$ & $\begin{array}{c}17179.6 \\
4\end{array}$ & 45.28 & 45.00 & 98.78 & 268.35 & 92.14 \\
\hline 13. & $\begin{array}{l}\text { Crude } \\
\text { protein } \\
\text { content (\%) }\end{array}$ & 5.56 & $\begin{array}{l}2.41- \\
8.90\end{array}$ & 2.56 & 2.47 & 28.81 & 28.29 & 96.43 & 3.18 & 57.23 \\
\hline 14. & $\begin{array}{l}\text { Grain yield } \\
(\mathrm{gm})\end{array}$ & 23.40 & $\begin{array}{l}12.62- \\
32.90\end{array}$ & 23.57 & 22.85 & 20.75 & 20.43 & 96.95 & 9.70 & 41.43 \\
\hline
\end{tabular}

Heritability in conjunction with genetic advance would give a more reliable index of selection value (Johnson et $a l .$, 1955). In the present study, except days to $50 \%$ flowering, all other highly heritable characters possessed high genetic advance as per cent of mean indicating that these characters were under the influence of additive gene action and hence selection for these characters could be effective in improving grain and stover yield in Pearl millet.

Based on $\mathrm{D}^{2}$ - statistics, 104 accessions were grouped into 12 clusters (Table 02). The maximum number of accessions (82) were included in cluster I, whereas clusters III -VII and IX - XII contained only 
one accession each, which would indicate extreme phenotypic performance in a positive or negative direction for one, or the other character. Cluster I included accessions from all sources, indicating that there was no association between pattern of clusters and geographical distribution of accessions, and this agrees with earlier findings of Thete et al. (1986), Joshi et al. (1988) and Wilson et al. (1990).

\section{Table 02: Composition of $\mathrm{D}^{2}$ clusters in pearl millet germplasm}

\begin{tabular}{|c|c|c|}
\hline $\begin{array}{l}\text { Cluster } \\
\text { number }\end{array}$ & $\begin{array}{l}\text { Number of } \\
\text { accessions }\end{array}$ & Accessions \\
\hline $\mathrm{I}$ & 82 & $\begin{array}{l}\text { GP 15074, GP 16271, IP 20381, GP 16026, GP 16016, GP } \\
\text { 18215, GP 18219, PT 5490, GP 16276, PT 5164/2, GP 15950, } \\
\text { IP 20350, PT 5139, PT 5376, PT 5164/1, GP 15974, } \\
\text { GP 16257, PT 5545, GP 15495, PT 5749, } \\
\text { GP 15072, PT 5896, PT 5142, PT 5276, } \\
\text { PT 5629/1, GP 15059, PT 5380, IP 3625, } \\
\text { PT 5359, IP 11354, PT 5259, IP 8600, } \\
\text { IP 20840, IP 20378, PT 5587, GP 15069, } \\
\text { IP 20379, PT 5222/, PT 5140, IP 3629, } \\
\text { PT 5382, PT 5407, PT 5821, IP 20334, } \\
\text { IP 20389, IP 5445, PT 5143, IP 20338, } \\
\text { PT 5701/1, PT 5647, PT 5577, PT 5542, } \\
\text { PT 5627, PT 5151, IP 20346, PT 5884, } \\
\text { GP 15953, IP 20349, IP 20273, PT 5491, } \\
\text { PT 5881, IP 20333, IP 20347, PT 5379, } \\
\text { PT 5386/1, PT 5387, PT 5217, GP 15958, } \\
\text { PT 5365, PT 5588, PT 5826, PT 5532, } \\
\text { PT 5684, PT 5165, PT 5790, PT 5580, } \\
\text { PT 5678, PT 5360, PT 5752, GP 16060, } \\
\text { GP 16021, GP 15076, }\end{array}$ \\
\hline II & 9 & $\begin{array}{l}\text { GP } 15189 \text {, IP 22269, GP 15073, GP 16239, GP 16023, GP } \\
\text { 15988, IP 19125, GP 16248, PT } 5136\end{array}$ \\
\hline III & 1 & PT 5362 \\
\hline IV & 1 & PT 5682 \\
\hline $\mathrm{V}$ & 1 & PT 5600 \\
\hline VI & 1 & PT 5665 \\
\hline VII & 1 & PT 5651 \\
\hline VIII & 4 & IP 8587, PT 5824, PT 5581, PT 5091 \\
\hline IX & 1 & PT 5652 \\
\hline $\mathrm{X}$ & 1 & GP 18222 \\
\hline XI & 1 & PT 5616 \\
\hline XII & 1 & GP 15071 \\
\hline
\end{tabular}


Considering the intra and inter-cluster distances (Table 03) the maximum inter-cluster distance was recorded between cluster XI and XII. It was noted that cluster XI was highly divergent from cluster II also. Other highly divergent cluster combination was cluster VIII with cluster XII and indicated that hybridization programme of genotypes belonging to these different divergent clusters might be used for exploitation of hybrid vigour and good recombinants.

Table 03: Inter and intra (diagonal) cluster average distance

\begin{tabular}{|c|c|c|c|c|c|c|c|c|c|c|c|c|}
\hline Clusters & I & II & III & IV & V & VI & VII & VIII & IX & $\mathrm{X}$ & XI & XII \\
\hline I & 43.29 & 75.30 & 66.44 & 66.81 & 64.81 & 80.80 & 63.97 & 90.18 & 59.77 & 63.83 & $\begin{array}{c}143.5 \\
4\end{array}$ & $\begin{array}{c}130.4 \\
9\end{array}$ \\
\hline II & & 42.13 & 123.52 & 120.80 & 114.89 & 132.79 & 117.33 & 142.82 & 88.22 & 68.98 & $\begin{array}{c}198.0 \\
5\end{array}$ & 78.98 \\
\hline III & & & 0.00 & 20.60 & 32.15 & 36.39 & 31.39 & 51.01 & 80.22 & 90.34 & 88.68 & $\begin{array}{c}178.1 \\
0\end{array}$ \\
\hline IV & & & & 0.00 & 23.79 & 28.16 & 36.23 & 53.84 & 85.46 & 80.61 & 86.37 & $\begin{array}{c}170.5 \\
0\end{array}$ \\
\hline $\mathrm{V}$ & & & & & 0.00 & 28.82 & 50.10 & 68.07 & 93.47 & 68.75 & 99.01 & $\begin{array}{c}161.0 \\
7\end{array}$ \\
\hline VI & & & & & & 0.00 & 46.60 & 57.09 & $\begin{array}{c}100.6 \\
4\end{array}$ & 88.06 & 77.70 & $\begin{array}{c}178.3 \\
0\end{array}$ \\
\hline VII & & & & & & & 0.00 & 47.83 & 64.28 & 93.28 & 93.41 & $\begin{array}{c}173.8 \\
5\end{array}$ \\
\hline VIII & & & & & & & & 45.01 & 85.54 & $\begin{array}{c}116.1 \\
1\end{array}$ & 73.83 & $\begin{array}{c}196.6 \\
6\end{array}$ \\
\hline IX & & & & & & & & & 0.00 & 99.75 & $\begin{array}{c}146.3 \\
2\end{array}$ & $\begin{array}{c}151.8 \\
1\end{array}$ \\
\hline $\mathrm{x}$ & & & & & & & & & & 0.00 & $\begin{array}{c}157.6 \\
6\end{array}$ & 93.82 \\
\hline XI & & & & & & & & & & & 0.00 & $\begin{array}{c}245.4 \\
0\end{array}$ \\
\hline XII & & & & & & & & & & & & 0.00 \\
\hline
\end{tabular}

The cluster means of various characters are represented in Table 04. Cluster XII, containing GP 15071 as the only genotype recorded the maximum mean for number of tillers, number of leaves, leaf weight, stover yield and grain yield. Cluster IX, having PT 5652, showed high mean values for leaf length and panicle length. Cluster II also showed maximum value for plant height and stem weight. Thus, the genotypes of outstanding mean performance from these clusters may be identified as potential parents and could be utilized in hybridizing programme of pearl millet. Their segregating progenies are also likely to yield good recombinants for economic traits. 
The relative contribution of different characters to genetic divergence showed that plant height contributed more $(35.34 \%)$ followed by leaf stem ratio $(29.00 \%)$ and leaf weight
$(21.86 \%)$ (Table 05). This indicated that these attributes should form the criteria for selection of parents for hybridization programme.

Table 04: Cluster means for fourteen characters in diverse accessions of pearl millet

\begin{tabular}{|c|c|c|c|c|c|c|c|c|c|c|c|c|c|c|}
\hline $\begin{array}{c}\text { Clust } \\
\text { ers }\end{array}$ & $\begin{array}{l}\text { Day } \\
\text { s to } \\
50 \% \\
\text { flow } \\
\text { erin } \\
\text { g }\end{array}$ & $\begin{array}{c}\text { Plan } \\
t \\
\text { heig } \\
\text { ht }\end{array}$ & $\begin{array}{c}\mathrm{Nu} \\
\mathrm{mbe} \\
\mathrm{r} \text { of } \\
\text { tiller } \\
\mathrm{s}\end{array}$ & $\begin{array}{c}\mathrm{Nu} \\
\mathrm{mbe} \\
\mathrm{r} \text { of } \\
\text { leav } \\
\text { es }\end{array}$ & $\begin{array}{c}\text { Leaf } \\
\text { leng } \\
\text { th }\end{array}$ & $\begin{array}{c}\text { Leaf } \\
\text { brea } \\
\text { dth }\end{array}$ & $\begin{array}{c}\text { Panic } \\
\text { le } \\
\text { lengt } \\
h\end{array}$ & $\begin{array}{c}\text { Stem } \\
\text { diamet } \\
\text { er }\end{array}$ & $\begin{array}{c}\text { Leaf } \\
\text { weig } \\
\text { ht }\end{array}$ & $\begin{array}{c}\text { Stem } \\
\text { weig } \\
\text { ht }\end{array}$ & $\begin{array}{l}\text { Leaf } \\
\text { stem } \\
\text { ratio }\end{array}$ & $\begin{array}{c}\text { Stove } \\
\mathrm{r} \\
\text { yield }\end{array}$ & $\begin{array}{c}\text { Crud } \\
\mathrm{e} \\
\text { protei } \\
\mathrm{n} \\
\text { conte } \\
\mathrm{nt}\end{array}$ & $\begin{array}{l}\text { Grain } \\
\text { yield }\end{array}$ \\
\hline I & $\begin{array}{c}52.4 \\
5\end{array}$ & $\begin{array}{c}165 \\
85\end{array}$ & 5.13 & $\begin{array}{c}33.1 \\
5\end{array}$ & $\begin{array}{c}48.3 \\
2\end{array}$ & 3.21 & 20.47 & 8.53 & 51.88 & $\begin{array}{c}225.3 \\
7\end{array}$ & 0.24 & $\begin{array}{c}277.2 \\
5\end{array}$ & 5.47 & 23.42 \\
\hline II & $\begin{array}{c}52.2 \\
2\end{array}$ & $\begin{array}{c}205 . \\
06\end{array}$ & 6.43 & $\begin{array}{c}52.8 \\
8\end{array}$ & $\begin{array}{c}47.1 \\
6\end{array}$ & 3.14 & 25.96 & 9.50 & 93.47 & $\begin{array}{c}469.6 \\
3\end{array}$ & 0.20 & $\begin{array}{c}563.0 \\
9\end{array}$ & 6.00 & 28.08 \\
\hline III & $\begin{array}{c}53.5 \\
0\end{array}$ & $\begin{array}{c}119 . \\
50\end{array}$ & 5.30 & $\begin{array}{c}20.9 \\
5\end{array}$ & $\begin{array}{c}39.8 \\
0\end{array}$ & 3.85 & 17.80 & 9.32 & 23.10 & 77.70 & 0.30 & $\begin{array}{c}100.8 \\
0\end{array}$ & 2.41 & 18.90 \\
\hline IV & $\begin{array}{c}54.0 \\
0\end{array}$ & $\begin{array}{c}104 . \\
55\end{array}$ & 4.45 & $\begin{array}{c}21.0 \\
0\end{array}$ & $\begin{array}{c}41.5 \\
0\end{array}$ & 2.55 & 15.10 & 9.58 & 40.45 & $\begin{array}{c}117.7 \\
5\end{array}$ & 0.35 & $\begin{array}{c}158.2 \\
0\end{array}$ & 6.71 & 16.78 \\
\hline V & $\begin{array}{c}52.5 \\
0\end{array}$ & $\begin{array}{c}101 \\
45\end{array}$ & 4.20 & $\begin{array}{c}33.1 \\
0\end{array}$ & $\begin{array}{c}33.0 \\
5\end{array}$ & 2.80 & 18.10 & 7.54 & 41.55 & $\begin{array}{c}161.8 \\
0\end{array}$ & 0.26 & $\begin{array}{c}203.3 \\
5\end{array}$ & 6.00 & 21.76 \\
\hline VI & $\begin{array}{c}54.5 \\
0\end{array}$ & $\begin{array}{c}91.4 \\
5\end{array}$ & 8.00 & $\begin{array}{c}39.4 \\
0\end{array}$ & $\begin{array}{c}32.5 \\
5\end{array}$ & 2.00 & 14.95 & 6.28 & 44.00 & $\begin{array}{c}116.0 \\
0\end{array}$ & 0.38 & $\begin{array}{c}160.0 \\
0\end{array}$ & 7.53 & 12.62 \\
\hline VII & $\begin{array}{c}53.0 \\
0\end{array}$ & $\begin{array}{c}137 \\
25\end{array}$ & 7.80 & $\begin{array}{c}40.8 \\
5\end{array}$ & $\begin{array}{c}49.9 \\
5\end{array}$ & 2.30 & 15.20 & 10.13 & 37.15 & 88.65 & 0.42 & $\begin{array}{c}125.8 \\
0\end{array}$ & 5.80 & 24.46 \\
\hline VIII & $\begin{array}{c}56.0 \\
0\end{array}$ & $\begin{array}{c}126 . \\
41\end{array}$ & 3.25 & $\begin{array}{c}17.6 \\
9\end{array}$ & $\begin{array}{c}41.6 \\
6\end{array}$ & 2.81 & 17.23 & 6.68 & 37.75 & 75.10 & 0.51 & $\begin{array}{c}112.8 \\
5\end{array}$ & 5.90 & 18.26 \\
\hline IX & $\begin{array}{c}52.5 \\
0\end{array}$ & $\begin{array}{c}201 \\
40\end{array}$ & 5.30 & $\begin{array}{c}35.0 \\
5\end{array}$ & $\begin{array}{c}64.9 \\
5\end{array}$ & 3.55 & 33.45 & 9.93 & 48.95 & $\begin{array}{c}106.4 \\
5\end{array}$ & 0.46 & $\begin{array}{c}155.4 \\
0\end{array}$ & 4.29 & 22.28 \\
\hline X & $\begin{array}{c}53.0 \\
0\end{array}$ & $\begin{array}{c}127 \\
75\end{array}$ & 5.65 & $\begin{array}{c}44.0 \\
5\end{array}$ & $\begin{array}{c}44.4 \\
0\end{array}$ & 2.65 & 18.80 & 8.18 & 96.15 & $\begin{array}{c}350.1 \\
0\end{array}$ & 0.28 & $\begin{array}{c}446.2 \\
5\end{array}$ & 5.72 & 26.26 \\
\hline XI & $\begin{array}{c}55.5 \\
0\end{array}$ & $\begin{array}{c}56.6 \\
5\end{array}$ & 3.15 & $\begin{array}{c}19.3 \\
5\end{array}$ & $\begin{array}{c}21.7 \\
5\end{array}$ & 2.10 & 12.80 & 8.60 & 32.35 & 51.85 & 0.63 & 84.20 & 7.29 & 13.19 \\
\hline XII & $\begin{array}{c}53.5 \\
0\end{array}$ & $\begin{array}{c}191 . \\
90\end{array}$ & 8.95 & $\begin{array}{c}61.1 \\
0\end{array}$ & $\begin{array}{c}53.4 \\
0\end{array}$ & 2.55 & 19.10 & 8.20 & $\begin{array}{c}163.0 \\
5\end{array}$ & $\begin{array}{c}439.0 \\
5\end{array}$ & 0.37 & $\begin{array}{c}602.1 \\
0\end{array}$ & 6.56 & 31.00 \\
\hline $\begin{array}{c}\text { Gene } \\
\text { ral } \\
\text { mean }\end{array}$ & $\begin{array}{c}53.5 \\
6\end{array}$ & $\begin{array}{c}135 . \\
77\end{array}$ & 5.63 & $\begin{array}{c}34.8 \\
8\end{array}$ & $\begin{array}{c}43.2 \\
1\end{array}$ & 2.79 & 19.08 & 8.54 & 59.15 & $\begin{array}{c}189.9 \\
5\end{array}$ & 0.37 & $\begin{array}{c}249.1 \\
1\end{array}$ & 5.81 & 21.42 \\
\hline
\end{tabular}


Table 05: Relative contribution of fourteen characters towards genetic divergence in 104 pearl millet germplam

\begin{tabular}{clcc}
\hline S1.No. & \multicolumn{1}{c}{ Characters } & $\begin{array}{c}\text { Number of times } \\
\text { ranked first }\end{array}$ & $\begin{array}{c}\text { Contribution } \\
(\%)\end{array}$ \\
\hline 1. & Days to 50\% flowering & 0 & 0.00 \\
2. & Plant height $(\mathrm{cm})$ & 1893 & 35.34 \\
3. & Number of tillers & 16 & 0.30 \\
4. & Number of leaves & 226 & 4.22 \\
5. & Leaf length (cm) & 214 & 4.00 \\
6. & Leaf breadth (cm) & 1 & 0.02 \\
7. & Panicle length (cm) & 137 & 2.56 \\
8. & Stem diameter (mm) & 75 & 1.40 \\
9. & Leaf weight (gm) & 1171 & 21.86 \\
10. & Stem weight (gm) & 4 & 0.07 \\
11. & Leaf stem ratio & 1553 & 29.00 \\
12. & Stover yield (gm) & 0 & 0.00 \\
13. & Crude protein content & 34 & 0.63 \\
14. & Grain yield (gm) & & \\
\hline
\end{tabular}

\section{CONCLUSION}

The study therefore, reveals that the selection based on all the characters except days to $50 \%$ flowering having high heritability coupled with greater genetic advance would be desirable for increasing grain and stover yield in pearl millet. Hybridization programme of genotypes belonging to II, VIII, XI and XII cluster might be used for exploitation of hybrid vigour. Their segregating progenies also likely to yield good recombinants for dual purpose.

\section{ACKNOWLEDGEMENT}

The first author is grateful to Council of Scientific and Industrial Research (CSIR), New Delhi, India for financial help. 


\section{References}

Berwal, K.K and I.S. Khairwal, (1997).genetic divergence in Pearl millet. International Sorghum and Millet Newsletter, 38, pp103-106.

Burton, G.W. (1952). Quantitative inheritance in grasses. Proceedings of $6^{\text {th }}$ International Grassland Congress, 1,pp 277-283.

Dangaria, C.J and S.D. Atara, (2004). GHB-558-A newly developed dual-purpose hybrid of pearl millet. Page 6 in Millet research and development - Future policy options in India, 11 Mar - 12 March 2004, Agricultural research station, Mandor, Jodhupur (Sharma, Y.K and Khairwal, I.S., eds). All India coordinated pearl millet improvement project.

Humphries, E.C. (1956). Mineral components and ash analysis. In : Modern Methods of plant Analysis. Vol I. (Eds.) K. Paech and M.V. Tracy, Springer verlag, Berlin, pp. 468-502.

Johnson, H.W.,. H.F Robinson, and R.E. Comstock, (1955). Estimation of genetic variability and environmental variability in soyabean. Agronomy Journal, 47,pp 314-318.

Joshi, R.P, G.S.Chauhan, and H.S. Yadav, (1988). Genetic divergence in Pearl millet. Experimental Genetics, 4, pp 16-28.

Lush, J.L. (1940). Intra-sire correlation and regression of offspring of dams as a method of estimating heritability of characters. Proceedings of American Society of Animal Production, 33, pp 293-301.

Mahalanobis, P.C. (1936). On the generalised distance in statistics. Journal of Genetics, 41,pp 159-193.

Rao, C.R. (1952). Advanced statistical method in Biometric Research. Edn. I. John Willey and Sons Inc., New York. pp. 390.

Suthamathi, P. and M.S Dorairaj, (1995). Variability, hertability and genetic advance in fodder pearl millet. Madras Agricultural Journal, 82(4), pp 238240.

Thete, R.Y., D.R. Bapal,. and S.D. Ugale, (1986). Heterosis in pearl millet. Current Research Report, 2,pp 16-25.

Vidyadhar, B., P Chand.,. S L Devi, I., M V S Reddy, and D.Ramachandraiah, (2004). Variability and correlation analysis for the yield attributes in pearl millet (Pennisetum glaucum (L.) R. Br.) germplasm. In : $3^{\text {rd }}$ National seminar on millet research and development - Future policy options in India. (Eds). Y.K. Sharma and I.S. Khairwal, Jodhupur, India, pp. 12.

Wilson, J.P., G.W.Burton, , J.D.Zongo, and I.O.Dicko, (1990). Diversity among pearl millet land races collected in Central Burkina Faso. Crop Science, 30, pp $40-43$. 